Noname manuscript No.

(will be inserted by the editor)

\title{
New Diversity Combining Receivers for Cooperative Multiplexing in Wireless Multiuser Relay Networks
}

Received: date / Accepted: date

\begin{abstract}
We propose two new diversity combining receivers that support cooperative multiplexing in two-hop wireless multiuser relay networks. Cooperative multiplexing has the potential to double the achievable throughput by allowing the base station (BS) and the relay station (RS) to transmit to different users at the same time in the second time slot of the half time division duplexed (TDD) relay transmission. This throughput improvement comes at a cost of performance degradation due to inter-user interference between the BS and the RS. To overcome this degradation, we propose two new receivers for the relay-link users: 1) cooperative multiplexing optimum combining (CMOC) and 2) cooperative multiplexing selection combining (CMSC). The proposed CMOC receiver combines the signals in the first and second time slot of the half TDD transmission such that the output signal-to-interference-plus-noise ratio (SINR) is maximized. The proposed CMSC receiver allows the relay-link user terminal to be active in only one of the two half TDD time slots. As such, CMSC offers power savings relative to CMOC. New insights are drawn from our exact closed-form expressions that we derive for the moment generation function, probability density function, and the cumulative distribution function of the output SINR. Based on these, we present new analytical expressions for the outage probability, symbol error rate, and achievable throughput. Our results show a 3.5 times improvement in the achievable throughput relative to the standard single-channel receiver in the high interference regime.
\end{abstract}

Keywords Cooperative Multiplexing · Optimum Combining · Selection Combining · Interference Channel

\section{Introduction}

The deployment of fixed relay stations (RS) in cellular networks is a low cost and low complexity solution to extend service coverage. The RS performs a

Address(es) of author(s) should be given 
similar role to the base station (BS) but with a subset of BS functions. In two-hop relay communications, half time-division duplex (TDD) is adopted in order for radio frequency devices to be easily implemented at low cost [1]. In standard half TDD, the RS receives data from the BS in the first time slot and forwards it to the relay-link user in the second time slot while the BS remains silent. This leads to a loss in data rates as two time slots are necessitated for interference-free transmission.

To allow the RS to receive and transmit in both time slots, multiple antennas were deployed at the RS in [2] and interference cancellation was applied to improve the data rate of the full-duplex system. In [3], two sophisticated resource handling schemes (antenna partitioning and antenna sharing) were proposed to improve the performance of full-duplex systems. Both schemes utilize multiple antennas at the RS to cancel inter-user interference.

When the RS is equipped with a single antenna, a cooperative multiplexing and scheduling scheme was considered to enhance the achievable throughput which simultaneously exploits spatial multiplexing and multiuser diversity gains [4-8]. Users are classified into two groups: direct-link users served by the BS and relay-link users served by the RS. Cooperative multiplexing increases the achievable throughput by allowing the BS to transmit to the direct-link user in the second time slot at the same time as the RS is transmitting to the relay-link user. ${ }^{1}$ An opportunistic scheduler is used to select users in each group to exploit the multiuser diversity $[10,11,15]$. This can be viewed as a multiplexed half TDD transmission. The potential is to double the throughput compared to standard half TDD transmissions subject to the ability to receive the RS signal in the presence of interference from the BS. Cooperative multiplexing with a single-channel receiver at the relay-link user was proposed in [4] to only detect the signal in the second time slot. In [4], the inter-user interference at the direct-link user and the relay-link user was considered to be sufficiently weak to be ignored. However, we note that if inter-user interference is not carefully handled at the receiver, cooperative multiplexing may result in a lower throughput than the non-multiplexed scenario with orthogonal time slots for the direct link user and the relay-link user.

In [5], the inter-user interference was canceled by distributed beamforming. In the proposed distributed beamforming, the BS and the RS coordinately generate transmit beams such that inter-user interference is pre-canceled. It comes at a cost of acquiring channel state information (CSI) at the BS. In $[6,7]$, the achievable throughput of cooperative multiplexing was improved by joint user and relay selection. However, this necessitates multiple RS to be deployed.

In [8], the inter-user interference at the direct-link user was canceled using a single RS and no CSI at the BS. To do so, the broadcast signal from the BS to the RS in the first time slot was utilized as prior knowledge of interference from the BS to the relay-link user in the second time slot. It was shown that the

\footnotetext{
1 This concept can be viewed within the framework of distributed multi-input/multioutput (MIMO) spatial multiplexing.
} 
interference at the direct-link user can be effectively eliminated, which in turn improves the achievable throughput. However, relay-link users still suffered from the interference caused by the signal from the BS to the direct-link user in the second time slot.

In this paper, we propose a complete solution in which the signals received in both the first and the second time slots of the half TDD transmission are combined to mitigate the inter-user interference at the relay-link user. This is in contrast to the standard single-channel receiver in [4] that only uses the signal from the RS in the second time slot. We reformulate the signals received in the first and the second time slots of multiplexed half TDD transmission as a multi-channel communication with co-channel interference. To do so, the first time slot is interference-free while the second time slot has a single interferer with the same channel magnitude as the desired signal in the first time slot. Our model is different from the standard multi-channel receiver with co-channel interference in [9]. This is due to the fact that in cooperative multiplexing the channel magnitude of desired signals and interfering signals are not independent. In this paper, we concentrate on decode-and-forward (DF) relaying that has been acknowledged in emerging standards such as 3GPP Long Term Evolution (LTE) and IEEE 802.16m [10, 11].

We first propose cooperative multiplexing optimum combining (CMOC) to overcome the performance degradation at the relay-link user due to inter-user interference. In the proposed CMOC, the signals received in both time slots in the multiplexed half TDD are weighted to maximize the output signal-tointerference-plus-noise ratio (SINR) at the relay-link user under the assumption that there is perfect decoding at the RS.

We then propose cooperative multiplexing selection combining (CMSC) by allowing the relay-link user to select only one time slot in the multiplexed half TDD transmission. In the proposed CMSC, the relay-link user measures the SINR of each time slot in the multiplexed half TDD during training periods and then chooses the time slot with the higher SINR for signal detection. The proposed CMSC allows the relay-link user terminal to only wake up in the selected time slot and go into sleep mode in the remaining time slot. This allows for power savings relative to CMOC.

In the two proposed diversity combining receivers, new insights are drawn from our exact closed-form expressions that we derive for the moment generation function (MGF), probability density function (PDF), and the cumulative distribution function (CDF) of the output SINR. Based on these, we further present new expressions for the achievable throughput, the outage probability, and the symbol error rate (SER) with $M$-PSK. In contrast to $[12,13]$ that considered optimum combining in the standard co-channel interference communication, we develop different analytical approaches for multiplexed half TDD transmission. For example, the MGF of the proposed CMOC can not be obtained by multiplying MGF of two single channel receivers as in [13] since channel magnitudes of the desired signal and the interfering signal are not independent. 


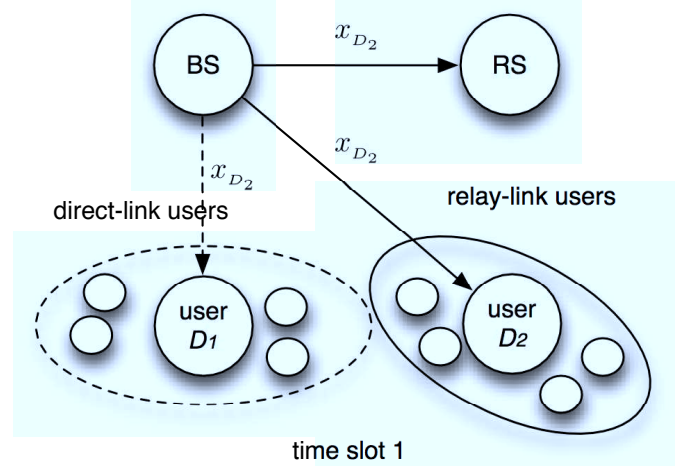

BS broadcasts data for user $D_{2}$

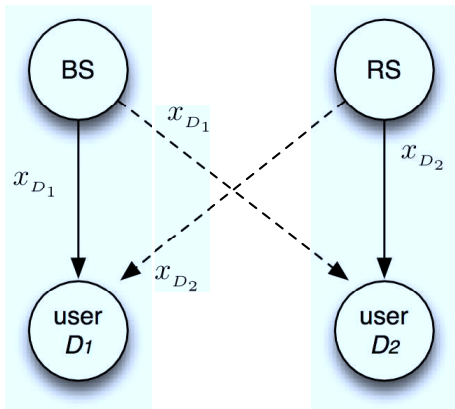

time slot 2

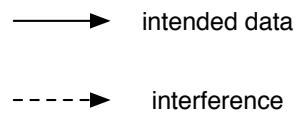

Fig. 1 System model for cooperative multiplexing and scheduling in wireless relay networks with $K=5$.

Through analysis and simulations, we evaluate the performance of the proposed two combining receivers and compare it with the standard single-channel receiver and the maximum ratio combining (MRC) receiver in [14]. Our simulations show that CMOC and CMSC outperform the single-channel receiver and the MRC receiver in all interference scenarios. Moreover, CMSC offers a superior performance over CMOC in terms of achievable throughput and the rate outage probability in the high interference regime. Furthermore, our proposed combining receivers show a 3.5 times improvement in the achievable throughput relative to the standard single-channel receiver and the MRC receiver in the high interference regime.

\section{Preliminaries}

\subsection{System Model}

We consider a single cell system with one BS and one RS. Both the BS and the RS are equipped with a single transmit/receive antenna. The users in the cell are classified as either direct-link users or relay-link users as shown in Fig.1. A single direct-link user is selected for transmission by the BS and a single relay-link user is selected for transmission by the RS.

We use multiplexed half TDD transmissions $[4,8]$ that consist of two adjacent time slots shorter than the channel coherent time. The transmission block is divided into two orthogonal time slots, and as with standard half TDD, each entity in the network (BS, RS, and users) can only transmit or receive in a given time slot [1]. In the first time slot, the BS transmits data for the relay-link user to the RS. Define $x_{k}$ as the transmit signal for the selected 
user $k$ with average power $E\left[x_{k} x_{k}^{*}\right]=P$. The signal from the BS to the RS in the first time slot, $y_{r}$ is represented by $y_{r}=h_{r} x_{k}+n_{r}$ where $h_{r}$ is the complex channel coefficient of the BS-RS link and $n_{r}$ is the additive white Gaussian noise (AWGN) with zero mean and variance $\sigma_{r}^{2}$. Here, ()$^{*}$ denotes the complex conjugate and the subscript $r$ indicates a variable related to the RS. In the second time slot, the RS forwards the signal received in the first time slot to the selected relay-link user. In the same second time slot, the BS also transmits to the selected direct-link user. In doing so, the BS and the RS constitute a virtual two-element antenna array used for spatial multiplexing and the two selected users are considered to be a distributed receive antenna array. As a result, the channel model in the second time slot can be described as a virtual MIMO channel.

We assume that CSI is measured according to a periodic channel training process whose period is defined by higher layer protocols. In each channel training process, the BS sends a pilot sequence in the first time slot and the RS sends a pilot sequence in the second time slot to allow users to estimate the CSI without inter-user interference. In this paper, we assume no CSI is available at the transmitter to perform joint signal processing except channel quality indicator for user scheduler.

We further consider DF relaying, where the RS decodes the received signal and then re-encodes it prior to transmission [1]. Denoting user $D_{1}$ as the selected direct-link user and user $D_{2}$ as the selected relay-link user, the received signal of the selected direct-link and relay-link users in the second time slot is represented by a $2 \times 2$ virtual MIMO channel as

$$
\mathbf{y}=\left[\begin{array}{l}
y_{D_{1}} \\
y_{D_{2}}
\end{array}\right]=\left[\begin{array}{ll}
h_{B D_{1}} & h_{R D_{1}} \\
h_{B D_{2}} & h_{R D_{2}}
\end{array}\right]\left[\begin{array}{l}
x_{D_{1}} \\
\hat{x}_{D_{2}}
\end{array}\right]+\left[\begin{array}{l}
n_{D_{1}} \\
n_{D_{2}}
\end{array}\right]
$$

where $h_{B k}$ and $h_{R k}$ are the complex channel coefficient from the BS and the RS to the selected user $k \in\left\{D_{1}, D_{2}\right\}$, respectively, and $n_{k}$ is an additive white Gaussian noise with $E\left[n_{k} n_{k}^{*}\right]=\sigma^{2}$ for all $k$. Also, $\hat{x}_{D_{2}}$ is the re-encoded relaying signal from the RS according to DF protocols. For convenience of presentation, we will omit the user index $k$ in $h_{B k}$ and $h_{R k}$ when it is clear that we are referring to the channel coefficients of given user $k$.

Users $D_{1}$ and $D_{2}$ (direct-link user and relay-link user) are selected via an opportunistic scheduler [15] in which radio resources are allocated to directlink user and relay-link user with the best reported channel quality. This user selection scheme is widely used to maximize sum data rate $[4,10,11,15]$. Throughout this paper, user $D_{2}$ is assumed to be selected from $K$ relay-link users. The BS and the RS do not perform any power control.

As DF relaying is employed, the achievable throughput of the relay-link user is computed as $[1,8]$

$$
\eta_{D_{2}}=\frac{1}{2} \log _{2}\left(1+\Upsilon\left(\gamma_{\mathrm{BR}}, h_{B D_{2}}, h_{R D_{2}}\right)\right)
$$

where $\gamma_{\mathrm{BR}}$ is the received SNR of the BS-RS link and $\Upsilon\left(\gamma_{\mathrm{BR}}, h_{B D_{2}}, h_{R D_{2}}\right)$ denotes the effective SINR at user $D_{2}$ which depends on the detection scheme 
employed. Here, the effective SINR is defined as the end-to-end SINR from the BS to the relay-link user that takes non-linear DF operations into account at the RS and signal combining at the relay-link user. Throughout this paper, we replace $\hat{x}_{D_{2}}$ in (1) with $x_{D_{2}}$ by assuming that BS transmits signals with the rate of $\eta_{D_{2}}$ in (2) using closed loop operations that guarantee error-free decoding. We now proceed to briefly outline the standard single-channel receiver and dual-channel maximal ratio combiner receiver which will be used in the comparison of our proposed cooperative multiplexing receivers.

\subsection{Standard Single-Channel Receiver}

In the standard single-channel receiver in [1], the relay-link user only detects the signal received in second time slot of multiplexed half TDD given as

$$
y_{D_{2}, 2}=h_{R} x_{D_{2}}+h_{B} x_{D_{1}}+n_{D_{2}, 2}
$$

In (3), the second term is the inter-user interference and degrades the performance in interference-limited environment.

Denoting the unfaded SNR as $\Omega \triangleq\left|x_{k}\right|^{2} / \sigma^{2}$ [1], the received SINR at the relay-link user with the standard single-channel receiver is expressed using (3) as

$$
\gamma_{D_{2}, \text { single }}=\frac{\left|h_{R}\right|^{2} \Omega}{\left|h_{B}\right|^{2} \Omega+1}
$$

and the achievable throughput of the relay-link user is

$$
\eta_{b, \text { single }}=\frac{1}{2} \log _{2}\left(1+\min \left(\gamma_{\mathrm{BR}}, \gamma_{D_{2}, \text { single }}\right)\right) .
$$

\subsection{Dual-Channel Maximum Ratio Combiner}

In the multiplexed half TDD transmission, the relay-link user can exploit the signal received in the first time slot to improve the performance when the interfering signal strength grows. The signal received in the first time slot is represented by

$$
y_{D_{2}, 1}=h_{B} x_{D_{2}}+n_{D_{2}, 1} .
$$

According to MRC [14], the detection of $x_{D_{2}}$ is based on the combined signal obtained as

$$
r=h_{B}^{*} y_{D_{2}, 1}+h_{R}^{*} y_{D_{2}, 2}
$$

where $y_{D_{2}, 2}$ is given in (3). The received SINR at the relay-link user with MRC is then expressed as

$$
\gamma_{D_{2}, \mathrm{mrc}}=\frac{\left(\left|h_{B}\right|^{2}+\left|h_{R}\right|^{2}\right)}{1 / \Omega+\frac{\left|h_{R}^{*} h_{B}\right|}{\left|h_{B}\right|^{2}+\left|h_{R}\right|^{2}}}
$$


and the achievable throughput of the relay-link user is

$$
\eta_{b, \mathrm{mrc}}=\frac{1}{2} \log _{2}\left(1+\min \left(\gamma_{\mathrm{RS}}, \gamma_{D_{2}, \mathrm{mrc}}\right)\right)
$$

\section{Cooperative Multiplexing Optimum Combining}

In this section, we propose CMOC by applying optimum combining for multichannel receivers with co-channel interference in [9] to detect signals at the relay-link user without prior knowledge of the BS-RS link. The performance of the proposed CMOC is analyzed by deriving the SER with $M$-PSK. Furthermore, new analytical expressions are derived for the PDF and CDF of the output SINR. In constructing the CMOC, we assume that there is perfect decoding at the RS since prior knowledge of the BS-RS link at the relay-link user requires additional signaling between the RS and relay-link users.

\subsection{Proposed CMOC}

At the relay-link user, the signals received in both time slots of the multiplexed half TDD is represented by a virtual MIMO communication model given by

$$
\begin{aligned}
\mathbf{y}_{D_{2}} & =\left[\begin{array}{l}
y_{2} \\
y_{1}
\end{array}\right]=\left[\begin{array}{cc}
h_{B} & h_{R} \\
0 & h_{B}
\end{array}\right]\left[\begin{array}{l}
x_{D_{1}} \\
x_{D_{2}}
\end{array}\right]+\left[\begin{array}{l}
n_{2} \\
n_{1}
\end{array}\right] \\
& =\left[\begin{array}{c}
h_{R} \\
h_{B}
\end{array}\right] x_{D_{2}}+\left[\begin{array}{c}
h_{B} \\
0
\end{array}\right] x_{D_{1}}+\mathbf{n} \\
& \triangleq \mathbf{c}_{D} x_{D_{2}}+\mathbf{c}_{I} x_{D_{1}}+\mathbf{n} .
\end{aligned}
$$

where $n_{1}$ and $n_{2}$ denote the additive Gaussian noise in the first and in the second time slot of half TDD transmission, respectively. The relay-link user combines the signals received in both time slots using a weight vector $\mathbf{w}$ such that the detection of $x_{D_{2}}$ is made on decision variable $\Lambda_{\mathrm{oc}}$ given by

$$
\Lambda_{\mathrm{oc}}=\mathbf{w}^{H} \mathbf{y}_{D_{2}}
$$

where $(\cdot)^{H}$ denotes the conjugate transpose operation.

The optimum combiner maximizes the average output SINR of the relaylink user given by

$$
\gamma \triangleq \frac{E_{s}\left|\mathbf{w}^{H} \mathbf{c}_{D}\right|^{2}}{\mathbf{w}^{H} \mathbf{R} \mathbf{w}}
$$

where $\mathbf{R}$ is the short term covariance matrix of the noise plus interference which is defined as

$$
\begin{aligned}
\mathbf{R} & =E\left[\left(\mathbf{c}_{I}+\mathbf{n}\right)^{H}\left(\mathbf{c}_{I}+\mathbf{n}\right)\right]=\tilde{\mathbf{R}}+\sigma^{2} \mathbf{I} \\
& =\left[\begin{array}{cc}
\left|h_{B}\right|^{2} E_{s}+\sigma^{2} & 0 \\
0 & \sigma^{2}
\end{array}\right]
\end{aligned}
$$


and $\tilde{\mathbf{R}}$ is defined as $E\left[\mathbf{c}_{I}^{H} \mathbf{c}_{I}\right]$. Here $\mathbf{I}$ denotes the $2 \times 2$ identity matrix. Without knowledge of the BS-RS link, the weighting vector $\mathbf{w}$ that maximizes the output SINR in (11) is given by [9]

$$
\mathbf{w}=\eta \mathbf{R}^{-1} \mathbf{c}_{D}=\left[\begin{array}{c}
\frac{h_{R}}{\left|h_{B}\right|^{2} E_{s}+\sigma^{2}} \\
\frac{h_{B}}{\sigma^{2}}
\end{array}\right]
$$

for an arbitrary constant $\eta$. Without loss of generality, we assume $\eta=1$ in this paper. The output SINR with the weighting vector $\mathbf{w}$ in (12) is then calculated as

$$
\gamma_{\mathrm{oc}}=E_{s} \mathbf{c}_{D}^{H} \mathbf{R}^{-1} \mathbf{c}_{D}=\frac{\left|h_{R}\right|^{2} E_{s}}{\left|h_{B}\right|^{2} E_{s}+\sigma^{2}}+\frac{\left|h_{B}\right|^{2} E_{s}}{\sigma^{2}} .
$$

We note that, when the interference term is extremely high, the output SINR of the CMOC is equal to direct transmission between the BS and the relaying-link user according to (12) indicates. On contrary, the output SINR of the single channel receiver in (4) becomes zeros in this case.

Recall that in DF relaying, the relay-link user detect the received signal correctly only when the signal from the BS is detected correctly at the RS. As such, the achievable throughput at the relay-link user based on (2) is expressed as

$$
\eta_{\mathrm{oc}}=\frac{1}{2} \log \left(1+\min \left(\gamma_{\mathrm{oc}}, \gamma_{\mathrm{BR}}\right)\right) .
$$

\subsection{Derived SER with $M$-PSK}

In this subsection, we derive the SER of CMOC at the relay-link user with Rayleigh fading in the BS-user and the RS-user link. In addition, we consider the more general case of Nakagami- $m$ fading in the BS-RS link. It is not straightforward to derive the SER of the proposed CMOC due to the fact that the two terms in (13) should be jointly considered since $\left|h_{B}\right|^{2}$ appears in both terms. As such, the MGF of $\gamma_{\mathrm{oc}}$ cannot be obtained simply by multiplying the individual MGF's of the signals received in the two time slots in multiplexed half TDD transmission.

The SER of CMOC with DF relaying and the weight vector defined in (12) is expressed as

$$
P_{e}=(1-\epsilon) P_{e}^{\mathrm{oc}}+\epsilon
$$

where $P_{e}^{\text {oc }}$ is the SER with respect to the output SINR $\gamma_{\text {oc }}$ in (13) and $\epsilon$ is the probability that the signal received in the RS is detected incorrectly. Assuming Nakagami- $m$ fading in the BS-RS link, $\epsilon$ with $M$-PSK modulation is computed as [16]

$$
\epsilon=\frac{1}{\pi} \int_{0}^{\Theta}\left(1+\frac{g_{M-\mathrm{PSK}} \bar{\gamma}_{\mathrm{BR}}}{2 m \sin ^{2} \theta}\right)^{-m} d \theta
$$


where $g_{M \text {-PSK }}=\sin ^{2} \frac{\pi}{M}$ and $\Theta=(M-1) \pi / M$. The expression in (15) can be written in a closed-form by using results in [16]. We note that, although we assumed perfect decoding at the RS to construct the CMOC weighting vector in (12), we co

Based on our formulation in (9), we note that the channel magnitude that contributes to the interference in the first term of (13) is the same as the channel magnitude of the desired signal in the second term of (13). As such, it is important to highlight that the results in $[12,13]$ cannot be directly used to derive the SER due to the fact that the channel magnitudes of the desired signal and the interfering signal are not independent.

We proceed to derive the SER of the proposed CMOC receiver with output SINR in (13) by applying the chain rule of conditional expectations

$$
P_{e}^{\mathrm{oc}}=E_{\left|h_{B}\right|^{2}}\left[\operatorname{Pr}\left(\left.\operatorname{error}\left|\gamma_{\mathrm{oc}}=\frac{\left|h_{R}\right|^{2} E_{s}}{\left|h_{B}\right|^{2} E_{s}+\sigma^{2}}+\frac{\left|h_{B}\right|^{2} E_{s}}{\sigma^{2}} ;\right| h_{B}\right|^{2}\right)\right] .
$$

Denote $x$ and $y$ as random variables representing $\left|h_{R}\right|^{2}$ and $\left|h_{B}\right|^{2}$, respectively. Using the MGF based approach to derive the SER in [16] and the property of conditional characteristic functions in [17], (16) is rewritten as

$$
\begin{aligned}
P_{e}^{\mathrm{oc}} & =E_{x}\left[\frac{1}{\pi} \int_{0}^{\Theta} M_{\gamma \mid x}\left(-\frac{g_{M-\mathrm{PSK}}}{\sin ^{2} \theta}\right) d \theta\right] \\
& =\int_{0}^{\infty} \frac{1}{\pi} \int_{0}^{\Theta} M_{\gamma \mid x}\left(-\frac{g_{M-\mathrm{PSK}}}{\sin ^{2} \theta}\right) d \theta f_{x}(x) d x
\end{aligned}
$$

where $f_{a}(\cdot)$ is the PDF the random variable $a$ and the conditional MGF $M_{\gamma \mid x}(s)$ is defined as

$$
M_{\gamma \mid x}(s)=\int_{0}^{\infty} \exp \left(s\left(\frac{x E_{s}}{y E_{s}+\sigma^{2}}+\frac{y E_{s}}{\sigma^{2}}\right)\right) f_{y}(y) d y .
$$

By changing the order of integration, (17) can be re-expressed as

$$
\begin{aligned}
P_{e}^{\mathrm{oc}} & =\frac{1}{\pi} \int_{0}^{\Theta} \int_{0}^{\infty} M_{\gamma \mid x}\left(-\frac{g_{M-\mathrm{PSK}}}{\sin ^{2} \theta}\right) f_{x}(x) d x d \theta \\
& \triangleq \frac{1}{\pi} \int_{0}^{\Theta} \Psi_{\mathrm{oc}}\left(-\frac{g_{M-\mathrm{PSK}}}{\sin ^{2} \theta}\right) d \theta
\end{aligned}
$$

where $\Psi_{\mathrm{oc}}(s)$ is defined as

$$
\Psi_{\mathrm{oc}}(s)=\int_{0}^{\infty} \int_{0}^{\infty} \exp \left(s\left(\frac{x E_{s}}{y E_{s}+\sigma^{2}}+\frac{y E_{s}}{\sigma^{2}}\right)\right) f_{x, y}(x, y) d y d x .
$$

We note that $\Psi_{\mathrm{oc}}(s)$ in (19) is not the standard joint MGF as defined in [14]. As such, we will refer to (19) as the modified joint MGF (MMGF). 
We can now derive the MMGF of $\gamma_{\text {oc }}$ in (19) as follows

$$
\begin{aligned}
\Psi_{\mathrm{oc}}(s) & =\int_{0}^{\infty}\left[\int_{0}^{\infty} \exp \left(s \frac{x E_{s}}{\sigma^{2}+y E_{s}}\right) f_{x}(x) d x\right] \exp \left(s \frac{y E_{s}}{\sigma^{2}}\right) f_{y}(y) d y \\
& =\int_{0}^{\infty} \frac{1}{1-s \frac{E_{s} \Omega_{R}}{y E_{s}+\sigma^{2}}} \exp \left(s \frac{y E_{s}}{\sigma^{2}}\right) \frac{1}{\Omega_{B}} \exp \left(-\frac{y}{\Omega_{B}}\right) d y \\
& =\frac{1}{\Omega_{B}} \int_{0}^{\infty}\left(1+\frac{s E_{s} \Omega_{R}}{y E_{s}+\sigma^{2}-s E_{s} \Omega_{R}}\right) \exp \left(-\left(\frac{1}{\Omega_{B}}-s \frac{E_{s}}{\sigma^{2}}\right) y\right) d y \\
& =\frac{1}{\Omega_{B}}\left[\frac{1}{\frac{1}{\Omega_{B}}-s \frac{E_{s}}{\sigma^{2}}}+s E_{s} \Omega_{R} \int_{0}^{\infty} \frac{\exp \left(-\left(\frac{1}{\Omega_{B}}-s \frac{E_{s}}{\sigma^{2}}\right) y\right)}{y E_{s}+\sigma^{2}-s E_{s} \Omega_{R}} d y\right]
\end{aligned}
$$

for $s<0$ where $\Omega_{B}=E\left[\left|h_{B}\right|^{2}\right], \Omega_{R}=E\left[\left|h_{R}\right|^{2}\right]$, and $h_{B}$ and $h_{R}$ are complex Gaussian in Rayleigh fading channels.

Using the integral identity in [18] and substituting $\Omega=E_{s} / \sigma^{2}$ we can derive the MMGF in (20) in a closed-form as

$$
\Psi_{\mathrm{oc}}(s)=\frac{1}{1-s \Omega_{B} \Omega}-s \frac{\Omega_{R}}{\Omega_{B}} \exp \left[\Phi_{\mathrm{oc}}(s)\right] \operatorname{Ei}\left(-\Phi_{\mathrm{oc}}(s)\right)
$$

where

$$
\Phi_{\mathrm{oc}}(s)=\left(\frac{1}{\Omega}-s \Omega_{R}\right)\left(\frac{1}{\Omega_{B}}-s \Omega\right)
$$

and $\operatorname{Ei}(x)$ is the exponential integral function defined in [19]. In (22), we can see that our derived MMGF is different from the MGF expressions for standard multi-channel receivers with co-channel interference in $[12,13]$ because the channel magnitude of the desired signal in the first time slot is the same as that of the interfering signal in the second time slot.

Note that, in computing $(21), \exp \left[\Phi_{\mathrm{oc}}(s)\right]$ in high SNR makes the numerical computation to be unstable. For $x>0$, the identity in [18]

$$
\operatorname{Ei}(-x)=-\exp (-x) \int_{0}^{1} \frac{d t}{x-\ln t}
$$

is utilized to avoid the numerical unstability in high SNR region by eliminating the term $\exp \left[\Phi_{\mathrm{oc}}(s)\right]$ in $(21)$. Consequently, $P_{e}^{\mathrm{oc}}$ that is obtained by substituting (21) into (18).

Finally, the SER of CMOC at the relay-link user is derived by substituting (18) into (14). We note that the derived SER expression is given as finite range single integrals of elementary mathematical functions. All the expressions can be easily evaluated using mathematical software such as MATLAB or Mathematica. Alternatively, (18) is well approximated using [13]

$$
\begin{aligned}
P_{e}^{\mathrm{oc}} \approx & \left(\frac{\Theta}{2 \pi}-\frac{1}{6}\right) \Psi_{\mathrm{oc}}\left(-g_{M-\mathrm{PSK}}\right)+\frac{1}{4} \Psi_{\mathrm{oc}}\left(-\frac{4 g_{M-\mathrm{PSK}}}{3}\right) \\
& +\left(\frac{\Theta}{2 \pi}-\frac{1}{4}\right) \Psi_{\mathrm{oc}}\left(-\frac{g_{M-\mathrm{PSK}}}{\sin ^{2} \Theta}\right) .
\end{aligned}
$$




\subsection{SINR Analysis for the Proposed CMOC}

In this subsection, we derive the CDF and the PDF of the output SINR at the relay-link user with the proposed CMOC receiver. By using the derived CDF and the PDF, the achievable throughput and the rate-outage probability is evaluated.

The effective SINR at the relay-link user with CMOC and DF relaying is the minimum of the received SNR at the RS and the output SINR of the combiner in (13). By using the property of function of two random variables in [17], the CDF of the effective SINR at the relay-link user is given as

$$
F_{D_{2}}^{\mathrm{oc}}(\gamma)=F_{\mathrm{oc}}(\gamma)+F_{\mathrm{BR}}(\gamma)-F_{\mathrm{oc}}(\gamma) F_{\mathrm{BR}}(\gamma)
$$

where $F_{\mathrm{oc}}(\gamma)$ denotes the $\mathrm{CDF}$ of the output SINR in (13) and $F_{\mathrm{BR}}(\gamma)$ is the $\mathrm{CDF}$ of the received $\mathrm{SNR}$ at the RS given as

$$
F_{\mathrm{BR}}(\gamma)=\frac{1}{\Gamma(m)} \gamma\left(m, \frac{m}{\bar{\gamma}}\right)
$$

for Nakagami- $m$ fading channels. Here, $\Gamma(m)$ is the gamma function defined as $\Gamma(m)=\int_{0}^{\infty} e^{-t} t^{m-1} d t$ and $\gamma(\alpha, x)$ is the incomplete gamma function defined as $\gamma(\alpha, x)=\int_{0}^{x} e^{-t} t^{\alpha-1} d t[19]$.

We derive a new CDF expression for $F_{\text {oc }}(\gamma)$ by defining a new random variable $z$ which is given by

$$
z=\frac{y}{x+\frac{1}{\Omega}}+x \Omega
$$

where $x$ and $y$ are re-defined two exponential random variables representing $\left|h_{B}\right|^{2}$ and $\left|h_{R}\right|^{2}$, respectively. As such, the CDF of the SINR at the output of the $\mathrm{OC}$ is evaluated as

$$
\begin{aligned}
F_{\mathrm{oc}}(\gamma) & =\operatorname{Pr}(z \leq \gamma)=\operatorname{Pr}\left(y \leq\left(x+\frac{1}{\Omega}\right)(\gamma-x \Omega)\right) \\
& =\int_{0}^{\infty} \operatorname{Pr}\left(y \leq\left(x+\frac{1}{\Omega}\right)(\gamma-x \Omega) \mid x\right) f_{x}(x) d x \\
& =\int_{0}^{\infty} \operatorname{Pr}\left(y \leq\left(x+\frac{1}{\Omega}\right)(\gamma-x \Omega)\right) f_{x}(x) d x
\end{aligned}
$$

where the last equality comes from the fact that $x$ and $y$ are independent. Since $x$ and $y$ are nonnegative, $\operatorname{Pr}\left(y \leq\left(x+\frac{1}{\Omega}\right)(\gamma-x \Omega)\right)$ in (24) is further analyzed as

$$
\operatorname{Pr}\left(y \leq\left(x+\frac{1}{\Omega}\right)(\gamma-x \Omega)\right)=\left\{\begin{array}{cc}
F_{y}\left(\left(x+\frac{1}{\Omega}\right)(\gamma-x \Omega)\right), & 0 \leq x \leq \frac{\gamma}{\Omega} \\
0, & x \geq \frac{\gamma}{\Omega}
\end{array}\right.
$$

where $F_{y}(y)$ is the CDF of $y$. 
By substituting $(25)$ into $(24), F_{\text {oc }}(\gamma)$ is calculated as

$$
\begin{aligned}
F_{\mathrm{oc}}(\gamma)= & \int_{0}^{\frac{\gamma}{\Omega}} F_{y}\left(\left(x+\frac{1}{\Omega}\right)(\gamma-x \Omega)\right) f_{x}(x) d x \\
= & F_{x}\left(\frac{\gamma}{\Omega}\right)-\frac{1}{\Omega_{B}} \int_{0}^{\frac{\gamma}{\Omega}} \exp \left(-\frac{1}{\Omega_{R}}\left(x+\frac{1}{\Omega}\right)(\gamma-x \Omega)\right) \exp \left(-\frac{x}{\Omega_{B}}\right) d x \\
= & 1-\exp \left(-\frac{1}{\Omega_{B}} \frac{\gamma}{\Omega}\right) \\
& -\frac{1}{\Omega_{B}} \underbrace{\int_{0}^{\frac{\gamma}{\Omega}} \exp \left(\frac{\Omega}{\Omega_{R}} x^{2}-\frac{\left((\gamma-1) \Omega_{B}+\Omega_{R}\right)}{\Omega_{R} \Omega_{B}} x-\frac{\gamma}{\Omega_{R} \Omega}\right) d x}_{\triangleq G(\gamma)}
\end{aligned}
$$

where $F_{x}(x)$ is the CDF of $x$. Using the integral identity in [18], $G(\gamma)$ in $(26)$ is computed according to

$$
G(\gamma)=\frac{1}{2} \sqrt{\frac{\pi}{a}} \exp \left(\frac{a c-b^{2}}{a}\right)\left[\operatorname{erfi}\left(\sqrt{a} \frac{\gamma}{\Omega}+\frac{b}{\sqrt{a}}\right)-\operatorname{erfi}\left(\frac{b}{\sqrt{a}}\right)\right]
$$

where erfi(x) is the imaginary error function [18] and

$$
\begin{aligned}
& a=\frac{\Omega}{\Omega_{R}}, \quad c=-\frac{\gamma}{\Omega_{R} \Omega}, \\
& b=-\frac{1}{2} \frac{\left((\gamma-1) \Omega_{B}+\Omega_{R}\right)}{\Omega_{R} \Omega_{B}} .
\end{aligned}
$$

The PDF of the output SINR is derived by differentiating (26) with respect to $\gamma$. Using the Leibniz's theorem for differentiation of a definite integral [19]

$$
\frac{d}{d \gamma} \int_{\psi(\gamma)}^{\varphi(\gamma)} f(x, \gamma) d x=\int_{\psi(\gamma)}^{\varphi(\gamma)} \frac{d}{d \gamma} f(x, \gamma) d x+f(\varphi(\gamma), \gamma) \frac{d \varphi(\gamma)}{d \gamma}-f(\psi(\gamma), \gamma) \frac{d \psi(\gamma)}{d \gamma}
$$

the PDF of the output SINR $f_{\text {oc }}(\gamma)$ is derived as

$$
\begin{aligned}
f_{\mathrm{oc}}(\gamma) & =\frac{d}{d \gamma} F_{\mathrm{oc}}(\gamma)=f_{x}\left(\frac{\gamma}{\Omega}\right)-\frac{1}{\Omega_{B}} \frac{d}{d \gamma} G(\gamma) \\
& =\frac{1}{\Omega_{R} \Omega_{B}} \int_{0}^{\frac{\gamma}{\Omega}}\left(x+\frac{1}{\Omega}\right) \exp \left(\frac{\Omega}{\Omega_{R}} x^{2}-\frac{\left((\gamma-1) \Omega_{B}+\Omega_{R}\right)}{\Omega_{R} \Omega_{B}} x-\frac{\gamma}{\Omega_{R} \Omega}\right) d x \\
& =\frac{1}{\Omega_{R} \Omega_{B}}\left[\int_{0}^{\frac{\gamma}{\Omega}} x \exp \left(\frac{\Omega}{\Omega_{R}} x^{2}-\frac{\left((\gamma-1) \Omega_{B}+\Omega_{R}\right)}{\Omega_{R} \Omega_{B}} x-\frac{\gamma}{\Omega_{R} \Omega}\right) d x+\frac{G(\gamma)}{\Omega}\right] \\
& =\frac{1}{\Omega_{R} \Omega_{B}}\left[\tilde{G}(\gamma)-\left(1-\frac{1}{\Omega}\right) G(\gamma)\right]
\end{aligned}
$$

where

$$
\tilde{G}(\gamma)=\frac{1}{2} \sqrt{\frac{\pi}{a}} \exp \left(\frac{a c-b^{2}}{a}\right) \operatorname{erfi}\left(\sqrt{a} \frac{\gamma}{\Omega}+\frac{b}{\sqrt{a}}\right)
$$


Using (26), (28), and order statistics in [20], we evaluate the average achievable throughput and the rate-outage probability of the CMOC with $K$ users as

$$
\bar{\eta}_{\mathrm{oc}}=\frac{1}{2} \int_{0}^{\infty} K \log _{2}(1+\gamma) f_{\mathrm{oc}}(\gamma) F_{\mathrm{oc}}^{K-1}(\gamma) d \gamma
$$

and

$$
P_{\text {out }}^{\text {oc }}(r)=\operatorname{Pr}\left(\frac{1}{2} \log (1+\gamma)<r\right)=F_{\text {oc }}^{K}\left(2^{2 r}-1\right) .
$$

\section{Cooperative Multiplexing Selection Combining}

The CMOC proposed in the previous section requires the signals received in both the first time slot and the second time slot to obtain the CMOC weighting vector in (12). In this section, we propose a new selection combining, named as CMSC, to overcome the performance degradation at the relay-link user by selecting only one time slot with the higher SINR in the multiplexed half TDD transmission. In doing so, the receiver is active in only one time slot of the multiplexed half TDD operation. As such, power savings is guaranteed by going into sleep mode in the remaining time slot. The proposed CMSC receiver offers power savings and improved battery lifetime at the cost of a performance degradation relative to CMOC. For the proposed CMSC, we derive the SER expression with $M$-PSK and useful statistical properties such as the PDF and the CDF of the output SINR, which are used to evaluate the achievable throughput and rate-outage probability.

\subsection{Proposed CMSC}

The key idea of our new receiver is to select the signal with the higher SINR between the time slots in the multiplexed half TDD. In the proposed CMSC, the relay-link user calculates the SINR during the training period as

$$
\begin{aligned}
& \gamma_{\mathrm{sc}, 1}=\left|h_{B}\right|^{2} \Omega \\
& \gamma_{\mathrm{sc}, 2}=\frac{\left|h_{R}\right|^{2}}{\left|h_{B}\right|^{2}+1 / \Omega} .
\end{aligned}
$$

The detection of $x_{D_{2}}$ in (9) is made on decision variable $\Lambda_{\mathrm{sc}}$ that is determined according to $\gamma_{\mathrm{sc}, 1}$ and $\gamma_{\mathrm{sc}, 2}$ given as

$$
\Lambda_{\mathrm{sc}}=\left\{\begin{array}{l}
y_{1}, \quad \gamma_{\mathrm{sc}, 1} \geq \gamma_{\mathrm{sc}, 2} \\
y_{2}, \quad \text { otherwise }
\end{array}\right.
$$

where $y_{1}$ and $y_{2}$ are defined in (9). 
The received SINR at the output of the proposed CMSC is then expressed as

$$
\gamma_{\mathrm{sc}}= \begin{cases}\gamma_{\mathrm{sc}, 1}, & \gamma_{\mathrm{sc}, 1} \geq \gamma_{\mathrm{sc}, 2} \\ \min \left\{\gamma_{\mathrm{sc}, 2}, \gamma_{\mathrm{BR}}\right\} & \text { otherwise }\end{cases}
$$

where the effective SINR based on $y_{2}$ is calculated as the minimum of the SNR at the RS $\left(\gamma_{\mathrm{BR}}\right)$ and the SINR at the relay-link user. Consequently, the achievable throuhgput is expressed as $\eta_{\mathrm{sc}}=\frac{1}{2} \log _{2}\left(1+\gamma_{\mathrm{sc}}\right)$.

\subsection{Derived SER with $M$-PSK Signals}

In this subsection, we derive the SER of CMSC. Denoting $\epsilon$ as the probability of detection error at the RS in (15) and $\kappa$ as $\operatorname{Pr}\left(\gamma_{\mathrm{sc}, 1} \geq \gamma_{\mathrm{sc}, 2}\right)$, the SER for CMSC with the proposed selection in (32) is expressed as

$$
P_{e}=\kappa P_{e}^{\mathrm{sc}, 1}+(1-\kappa)\left[(1-\epsilon) P_{e}^{\mathrm{sc}, 2}+\epsilon\right]
$$

where $P_{e}^{\mathrm{sc}, 1}$ is the SER when the receiver is activated in the first time slot and $P_{e}^{\mathrm{sc}, 2}$ is the SER when the receiver is activated in the second time slot.

We begin by deriving $\operatorname{Pr}\left(\gamma_{\mathrm{sc}, 1} \geq \gamma_{\mathrm{sc}, 2}\right)$ as follows. Defining two random variables $x$ and $y$ that represent each realization of $\left|h_{B}\right|^{2} \Omega$ and $\left|h_{R}\right|^{2} \Omega$, respectively, $\operatorname{Pr}\left(\gamma_{\mathrm{sc}, 1} \geq \gamma_{\mathrm{sc}, 2}\right)$ is computed as

$$
\begin{aligned}
\operatorname{Pr}\left(\gamma_{\mathrm{sc}, 1} \geq \gamma_{\mathrm{sc}, 2}\right) & =\operatorname{Pr}\left(x>\frac{y}{x+1}\right)=\int_{0}^{\infty} \operatorname{Pr}(y<x(x+1) \mid x) f_{x}(x) d x \\
& =\int_{0}^{\infty} F_{y}(x(x+1)) f_{x}(x) d x
\end{aligned}
$$

where $x$ and $y$ are random variables representing $\left|h_{B}\right|^{2}$ and $\left|h_{R}\right|^{2}$, respectively. In Rayleigh fading, $x$ and $y$ are exponential random variables with mean $\bar{\gamma}_{B}=$ $\Omega_{B} \Omega$ and $\bar{\gamma}_{R}=\Omega_{R} \Omega$, respectively. Thus, (35) is manipulated as

$$
\begin{aligned}
\operatorname{Pr}\left(\gamma_{\mathrm{sc}, 1} \geq \gamma_{\mathrm{sc}, 2}\right) & =\frac{1}{\bar{\gamma}_{B}} \int_{0}^{\infty}\left(1-\exp \left(-\frac{x^{2}+x}{\bar{\gamma}_{R}}\right)\right) \exp \left(-\frac{x}{\bar{\gamma}_{B}}\right) d x \\
& =1-\frac{1}{\bar{\gamma}_{B}} \int_{0}^{\infty} \exp \left(-\frac{x^{2}}{\bar{\gamma}_{B}}-\left(\frac{1}{\bar{\gamma}_{B}}+\frac{1}{\bar{\gamma}_{R}}\right) x\right) d x
\end{aligned}
$$

Using the integral identity in [18]

$$
\int_{0}^{\infty} \exp \left(-\frac{x^{2}}{4 \beta}-\alpha x\right) d x=\sqrt{\pi \beta} \exp \left(\beta \gamma^{2}\right)[1-\operatorname{erf}(\alpha \sqrt{\beta})] \text { for } \beta>0
$$

where $\operatorname{erf}(x)$ is the error function, (36) is evaluated by substituting $\alpha=$ $\left(\frac{1}{\bar{\gamma}_{B}}+\frac{1}{\bar{\gamma}_{R}}\right)$ and $\beta=\bar{\gamma}_{B} / 4$ into (37). 
The SER when the first time slot in the multiplexed half-duplex TDD is selected to detect user signals is obtained as

$$
P_{e}^{\mathrm{sc}, 1}=\frac{1}{\pi} \int_{0}^{\Theta} M_{\mathrm{sc}, 1}\left(-\frac{g_{M-\mathrm{PSK}}}{\sin ^{2} \theta}\right) d \theta
$$

where $M_{\mathrm{sc}, 1}(s)$ is equal to the MGF of Rayleigh fading channels given as [16]

$$
M_{\mathrm{sc}, 1}(s)=\frac{1}{1-s \bar{\gamma}_{B}} .
$$

Utilizing the method used in (18), the SER when the second time slot in the multiplexed half-duplex TDD is selected is obtained as

$$
P_{e}^{\mathrm{sc}, 2}=\frac{1}{\pi} \int_{0}^{\Theta} \Psi_{\mathrm{sc}, 2}\left(-\frac{g_{M-\mathrm{PSK}}}{\sin ^{2} \theta}\right) d \theta
$$

where the MMGF of $\gamma_{\mathrm{sc}, 2}$ is derived as

$$
\begin{aligned}
\Psi_{\mathrm{sc}, 2}(s) & =\int_{0}^{\infty} \int_{0}^{\infty} \exp \left(s \frac{y}{1+x}\right) \frac{1}{\bar{\gamma}_{R}} \exp \left(-\frac{1}{\bar{\gamma}_{R}} y\right) d y \frac{1}{\bar{\gamma}_{B}} \exp \left(-\frac{1}{\bar{\gamma}_{B}} x\right) d x \\
& =\int_{0}^{\infty} \frac{1+x}{1+x-s \bar{\gamma}_{R}} \frac{1}{\bar{\gamma}_{B}} \exp \left(-\frac{1}{\bar{\gamma}_{B}} x\right) d x \\
& =1+\frac{1}{\bar{\gamma}_{B}} \int_{0}^{\infty} \frac{s \bar{\gamma}_{R}}{1+x-s \bar{\gamma}_{R}} \exp \left(-\frac{1}{\bar{\gamma}_{B}} x\right) d x
\end{aligned}
$$

By using the integral identity in [18], we derive (40) in a closed-form as

$$
\Psi_{\mathrm{sc}, 2}(s)=1-\frac{\bar{\gamma}_{R}}{\bar{\gamma}_{B}} \exp \left(\frac{1-s \bar{\gamma}_{R}}{\bar{\gamma}_{B}}\right) \operatorname{Ei}\left(-\frac{1-s \bar{\gamma}_{R}}{\bar{\gamma}_{B}}\right) .
$$

Finally, by applying (15), (37), (38) and (39) to (34), the SER of the proposed CMSC is obtained. We note that the derived expression for SER is given as finite range single integrals of elementary mathematical functions that can be evaluated efficiently using standard mathematical software.

\subsection{SINR Analysis for the Proposed CMSC}

Under CMSC, we derive the CDF and the PDF of the output SINR at the relay-link user. According to the selection scheme in (32), the received SINR at the output of the CMSC in (33) can be rewritten as

$$
\gamma_{\mathrm{sc}}=\max \left(\gamma_{\mathrm{sc}, 1}, \min \left\{\gamma_{\mathrm{sc}, 2}, \gamma_{\mathrm{BR}}\right\}\right) .
$$

Define $\gamma_{\mathrm{sc}, \mathrm{df}}$ as a random variable representing the received SINR at the second time slot of half TDD given by $\min \left\{\gamma_{\mathrm{sc}, 2}, \gamma_{\mathrm{BR}}\right\}$. Using the property of function 
of two random variables in [17], the CDF of the effective SINR at the relay-link user when the receiver is activated in the second time slot is obtained as

$$
F_{\mathrm{sc}, \mathrm{df}}(\gamma)=F_{\mathrm{BR}}(\gamma)+F_{\mathrm{sc}, 2}(\gamma)-F_{\mathrm{BR}}(\gamma) F_{\mathrm{sc}, 2}(\gamma)
$$

where $F_{\mathrm{BR}}(\gamma)$ and $F_{\mathrm{sc}, 2}(\gamma)$ are $\mathrm{CDF}$ of $\gamma_{\mathrm{BR}}$ and $\gamma_{\mathrm{sc}, 2}$, respectively.

Then the CDF of the proposed CMSC is derived by

$$
F_{\mathrm{sc}}(\gamma)=F_{\mathrm{sc}, 1}(\gamma) F_{\mathrm{sc}, \mathrm{df}}(\gamma
$$

where $F_{\mathrm{sc}, \mathrm{df}}(\gamma)$ is the CDF of $\gamma_{\mathrm{sc}, \mathrm{df}}$. The PDF of the received SINR with CMSC is then obtained by evaluating the first derivative of (43) given as

$$
\begin{aligned}
f_{\mathrm{sc}}(\gamma)=\frac{d F_{\mathrm{sc}}(\gamma)}{d \gamma}= & F_{\mathrm{sc}, 1}(\gamma)\left[f_{\mathrm{BR}}(\gamma)+f_{\mathrm{sc}, 2}(\gamma)-f_{\mathrm{BR}}(\gamma) F_{\mathrm{sc}, 2}(\gamma)-F_{\mathrm{BR}}(\gamma) f_{\mathrm{sc}, 2}(\gamma)\right] \\
& +f_{\mathrm{sc}, 1}(\gamma)\left[F_{\mathrm{BR}}(\gamma)+F_{\mathrm{sc}, 2}(\gamma)-F_{\mathrm{BR}}(\gamma) F_{\mathrm{sc}, 2}(\gamma)\right]
\end{aligned}
$$

Denoting $x$ and $y$ as random variables representing each realization of $\left|h_{B}\right|^{2} \Omega$ and $\left|h_{R}\right|^{2} \Omega$, respectively, $\gamma_{\mathrm{sc}, 2}$ is represented by $\gamma_{\mathrm{sc}, 2}=y /(x+1)$. We derive the $\mathrm{CDF}$ of $\gamma_{\mathrm{sc}, 2}$ in (42) in closed-form as

$$
\begin{aligned}
F_{\gamma_{\mathrm{sc}, 2}}(\gamma) & =\int_{0}^{\infty} F_{y}((1+x) \gamma) f_{x}(x) d x \\
& =\int_{0}^{\infty}\left[1-\exp \left(-\frac{(1+x) \gamma}{\bar{\gamma}_{R}}\right)\right] \frac{1}{\bar{\gamma}_{B}} \exp \left(-\frac{x}{\bar{\gamma}_{B}}\right) d x \\
& =1-\frac{1}{\bar{\gamma}_{B}} \exp \left(-\frac{\gamma}{\bar{\gamma}_{R}}\right) \int_{0}^{\infty} \exp \left[-\left(\frac{\gamma}{\bar{\gamma}_{R}}+\frac{1}{\bar{\gamma}_{B}}\right) x\right] d x \\
& =1-\exp \left(-\frac{\gamma}{\bar{\gamma}_{R}}\right) \frac{1}{\frac{\bar{\gamma}_{B}}{\bar{\gamma}_{R}} \gamma+1} .
\end{aligned}
$$

Also, the PDF of $\gamma_{\mathrm{sc}, 2}$ is derived by taking the first derivative of (45) given as

$$
\begin{aligned}
f_{\gamma_{\mathrm{sc}, 2}}(\gamma) & =\frac{d F_{\gamma_{\mathrm{sc}, 2}(\gamma)}}{d \gamma}=\frac{1}{\bar{\gamma}_{R}} \exp \left(-\frac{\gamma}{\bar{\gamma}_{R}}\right) \frac{1}{\frac{\overline{\bar{\gamma}}_{B}}{\overline{\bar{\gamma}}_{R}}+1}-\frac{\bar{\gamma}_{B}}{\bar{\gamma}_{R}}\left(\frac{\bar{\gamma}_{B}}{\bar{\gamma}_{R}} \gamma+1\right)^{-2} \exp \left(-\frac{\gamma}{\bar{\gamma}_{R}}\right) \\
& =\frac{1}{\bar{\gamma}_{R} \bar{\gamma}_{B}} \exp \left(-\frac{\gamma}{\bar{\gamma}_{R}}\right)\left(\frac{\gamma}{\bar{\gamma}_{R}}+\frac{1}{\bar{\gamma}_{B}}\right)^{-2}\left(\frac{\gamma}{\bar{\gamma}_{R}}+\frac{1}{\bar{\gamma}_{B}}+1\right)
\end{aligned}
$$

Using (43)-(44) and order statistics in [20], we evaluate the average achievable throughput and the rate outage probability of the proposed CMSC with $K$ users as

$$
\bar{\eta}_{\mathrm{sc}}=\frac{1}{2} \int_{0}^{\infty} K \log _{2}(1+\gamma) f_{\mathrm{sc}}(\gamma) F_{\mathrm{sc}}^{K-1}(\gamma) d \gamma
$$

and

$$
P_{\text {out }}^{\mathrm{sc}}(r)=\operatorname{Pr}\left(\frac{1}{2} \log (1+\gamma)<r\right)=F_{\mathrm{sc}}^{K}\left(2^{2 r}-1\right)
$$




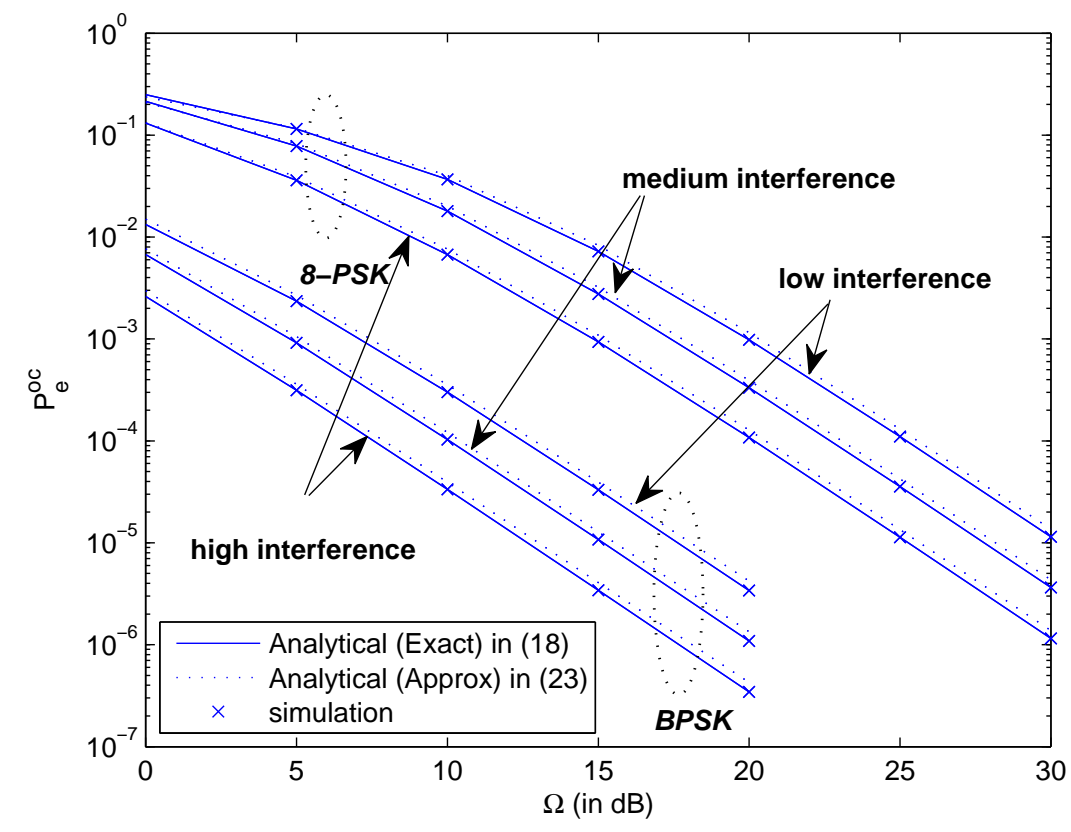

Fig. 2 SER of CMOC in (12) as a function of unfaded SNR $\Omega$; comparison between analytical and simulation results for different modulation schemes.

\section{Numerical Results}

In this section, we present numerical examples to validate the preceding analysis for our proposed diversity combining receivers. We highlight their performance advantage relative to the standard single channel receiver and the MRC receiver for three interference levels of $\rho=\Omega_{B} / \Omega_{R}$. Specifically, we set $\rho=$ $-10 \mathrm{~dB},-20 \mathrm{~dB}$ and $-30 \mathrm{~dB}$, which are referred to as high interference, medium interference, and low interference, respectively [8]. In this section, we focus on the performance with a single relay-link user since the statistics of multiuser diversity gain with the opportunistic scheduler can be directly obtained for CMOC using (29)-(30), and for CMSC using (47)-(48).

Fig. 2 plots the analytical and Monte-Carlo simulated SER curves for CMOC in (12) versus the unfaded SNR $\Omega$. We assume an error-free BS-RS link with $\epsilon=0$ in (14) to focus on verifying derived analytical expressions. In the figure, we present results for BPSK $(M=2)$ and 8-PSK $(M=8)$. The exact analytical curves are generated using (21) and (18), and the approximate curves are generated using (23). We see that, in all cases, the exact SER curves match well with the simulation results. The approximate curves also accurately estimate the exact results with reduced computational complexity. In the figure, we see that the SER improves with increasing $\rho$. This is due to 


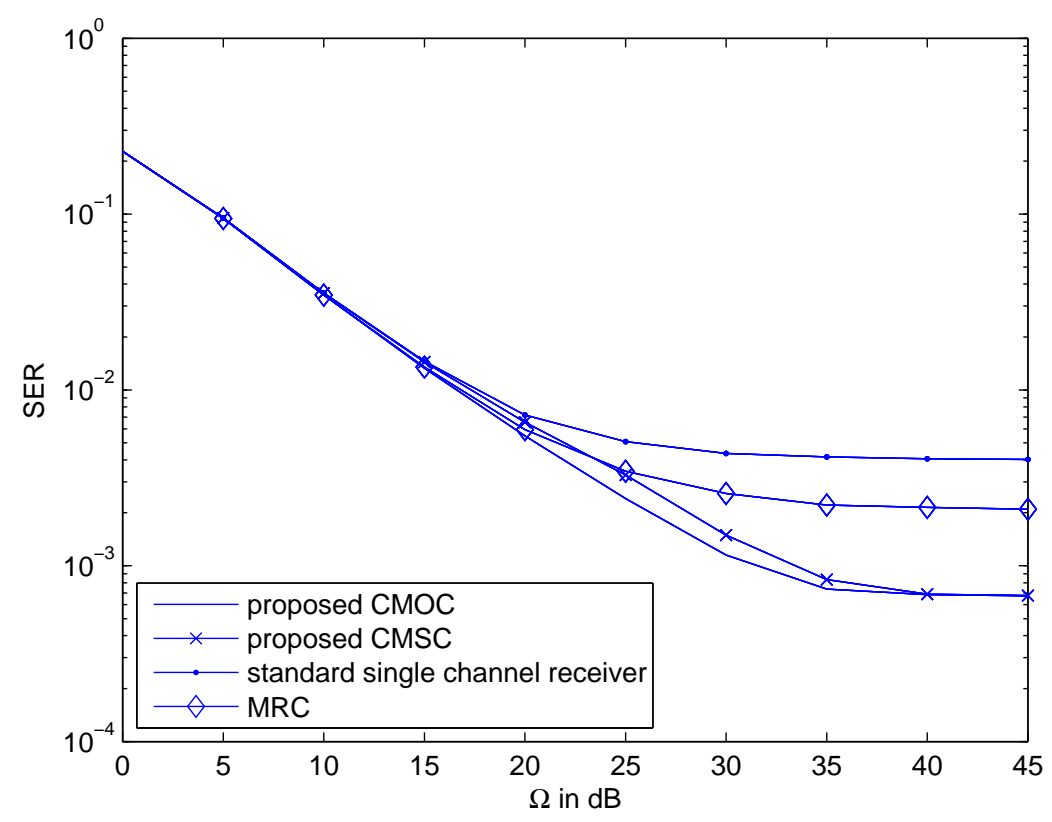

Fig. 3 SER comparison at the relay-link user in low interference.

the fact that increasing $\rho$ corresponds to increasing the signal strength from the BS in the first time slot.

Figs. 3 to 5 plots the SER of CMOC, CMSC, MRC, and the single channel receiver. In the figures, we assume the average received SNR of the BS-RS link to be $40 \mathrm{~dB}$ that corresponds to $\epsilon=6.7 \times 10^{-4}$ in (14) and (34). We see that CMOC achieves the best SER in all three interference scenarios. The proposed CMOC and CMSC outperform MRC and the single channel receiver by properly exploiting the signal received in the first time slot. We observe that the performance of CMSC is slightly inferior to CMOC. However, in the proposed CMSC, the relay-link user needs to wake up in only one time slot in the multiplexed half TDD transmission. We note that an error floor is observed due to the fact that the SER is restricted by DF relaying in the BS-RS link in the high SNR regime.

Fig. 6 plots the achievable throughput of CMOC, CMSC, MRC, and the single channel receiver. The achievable throughput of CMOC and CMSC is also evaluated using (29) and (47), respectively. For comparison, we include the achievable throughput with non-multiplexed two-hop relay. The non-multiplexed two-hop relay carries out interference-free transmission by dividing one transmission block into two orthogonal sub-blocks in which each sub-block is exclusively used for either the direct-link user or the relay-link user. When single channel receiver is employed in the cooperative multiplexing, the achievable throughput is shown to be worse than the non-multiplexed 


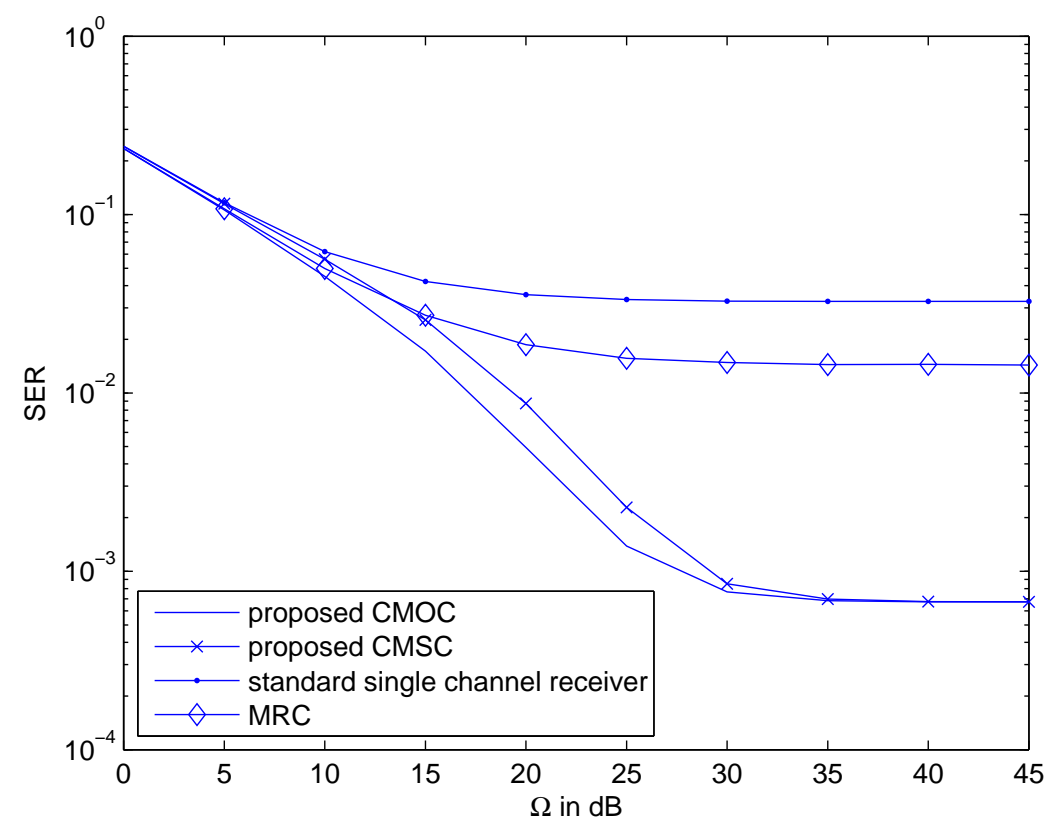

Fig. 4 SER comparison at the relay-link user in medium interference.

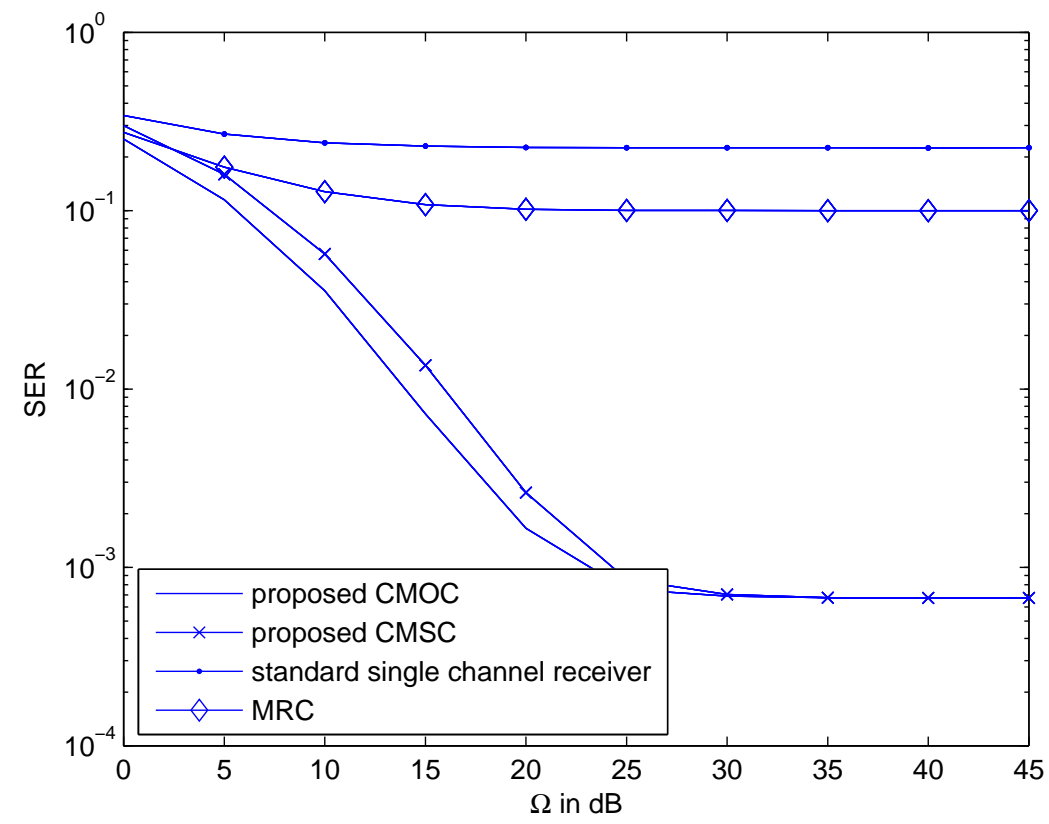

Fig. 5 SER comparison at the relay-link user in high interference. 


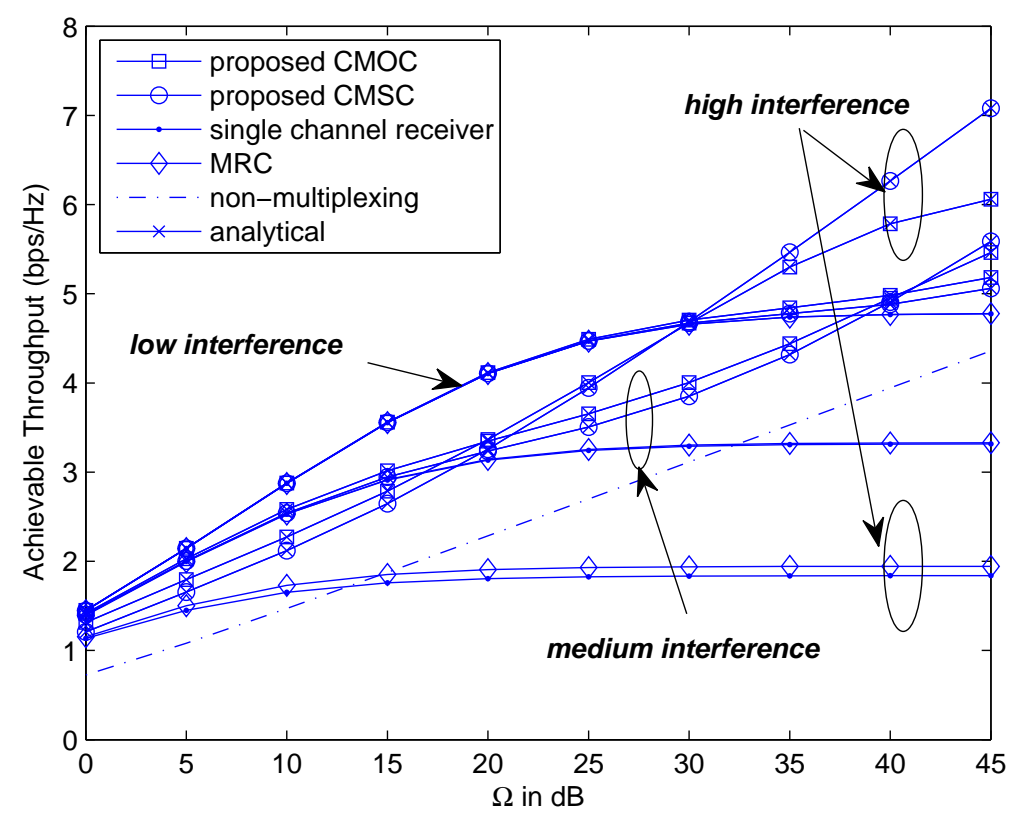

Fig. 6 Achievable throughput comparison as a function of unfaded SNR $\Omega$.

two-hop relay in the high interference regime. On contrary, our proposed combining receiver always exhibits better achievable throughput than the nonmultiplexed two-hop relay in interference levels. In this figure, the performance of the CMSC outperforms the CMOC in high SNR region. This is due to the fact that, at high SNR, the BS-RS link dominates the achievable throughput of the CMOC by the DF relaying operation. In other words, the proposed CMSC will show better performance than the CMOC receiver if capacity approaching channel codes such as turbo-code or low-density parity check code are employed. But we will leave this as future research. We see that the achievable throughput of the MRC and the single channel receiver decreases with increasing interference from the BS. In the figure, we see that CMOC and CMSC provide up to 3.5 times improvement in the achievable throughput relative to the single channel receiver up to $60 \%$ improvement to the non-multiplexed two-hop relay in the high interference regime.

Finally, in Fig. 7, we see the impact of the signal strength from the BS on the rate-outage probability of CMOC and CMSC derived in (30) and (48), respectively. We first verify that the performance of the three receivers is almost the same at $\rho<-25 \mathrm{~dB}$. As the signal strength from the BS increases, the rate-outage performance of CMOC improves monotonically with an outage floor at $\rho>-8 \mathrm{~dB}$. The floor arises due to the fact that the BS-RS link dominates the overall performance in the high interference regime. Interestingly, we find that CMSC outperforms CMOC at high $\rho>-8 \mathrm{~dB}$. This is because 


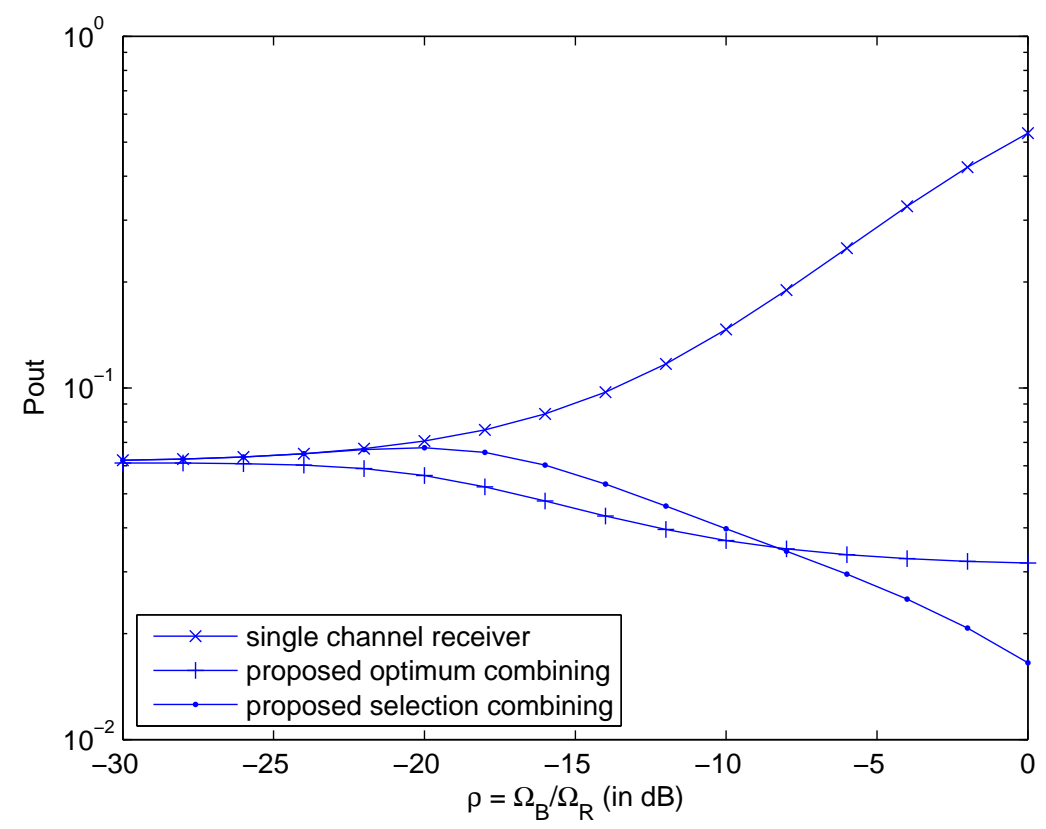

Fig. 7 Comparison of rate outage probability with respect to signal strength from the BS and fixed $\Omega_{R}$.

CMSC is not restricted by incorrect detection in the BS-RS link in the high interference regime.

\section{Conclusion}

We proposed two new diversity combining receivers, namely CMOC and CMSC, that support cooperative multiplexing in two-hop multiuser relay networks. The proposed receivers allow the BS and the RS to transmit to different users at the same time and effectively mitigate the inter-user interference at the relay-link users. By exploiting the signals received in both time slots of the cooperative multiplexed half duplex TDD, we demonstrated that the SER performance and the achievable throughput of CMOC and CMSC were notably higher relative to the standard single-channel receiver in [8]. For the proposed diversity combining receivers, we derived SER expressions with M-PSK and useful statistical properties such as the PDF, CDF, and MGF of the output SINR. Based on these, we analyzed the achievable throughput and rate-outage probability. A 3.5 times improvement in the achievable throughput relative to the standard single-channel receiver was demonstrated. 


\section{References}

1. J. Nicolas Laneman and David N. C. Tse and Gregory W. Wornell, Cooperative Diversity in Wireless Networks: Efficient Protocols and Outage Behavior, IEEE Transactions on Information Theory, Vol. 50, No. 12, pp. 3062-3080 (2004)

2. T. Riihonen and S. Werner and R. Wichman, Spatial Loop Interference Suppression in Full-duplex MIMO Relays, in Proc. Asilomar Conf. Signals, Systems and Computers, pp. 1508-1512 (2009)

3. H. Ju and E. Oh and D. Hong, Improving Efficiency of Resource Usage in Two-hop Full Duplex Relay Systems Based on Resource Sharing and Interference Cancellation, IEEE Transactions on Wireless Communications, Vol. 8, No. 8, pp. 3933-3938 (2009)

4. Yi Shi and Wei Zhang and Khaled Ben Letaief, Improving Efficiency of Resource Usage in Two-hop Full Duplex Relay Systems Based on Resource Sharing and Interference Cancellation, in Proc. IEEE International Conference on Communications (ICC'08)(2008)

5. Jinho Choi, Distributed Beamforming with Consensus Algorithm for Cooperative Relay Networks, IEEE Commuications Letters, Vol. 15, No. 4, pp. 368-370 (2011)

6. Jinho Choi, Distributed Beamforming with Consensus Algorithm for Cooperative Relay Networks, IEEE Commuications Letters, Vol. 15, No. 4, pp. 368-370 (2011)

7. D. K. Verma and S. Prakriya, Performance of a Cooperative Multipexing Sheme with Opportunistic User and Relay Selection over Rayleigh Fading Channels, accepted in EURASIP Journal on Wireless Communications and Networking (2012)

8. C. K. Sung and I. B. Collings, Cooperative Multiplexing with Interference Suppression in Multiuser Wireless Relay Networks, IEEE Transactions on Wireless Communications, vol. 9, pp. 2528-2538 (2010)

9. J. Winters, Optimum Combining in Digital Mobile Radio with Cochannel Interference, IEEE Journal on Selected Areas in Communications, vol. 2, pp. 528-539 (1984).

10. ETSI TS 136211 V9.0.0 E-UTRA; Physical Channels and Modulation (3GPP TS 36.211 Release10). ETSI (2011).

11. IEEE Std 802.16m-2011, IEEE Standard for Local and metropolitan area networks Part 16: Air Interface for Fixed Broadband Wireless Access Systems-Amendment 3: Advanced Air Interface (2011).

12. M. Chiani, M. Win, A. Zanella, R. K. Mallik, and J. Winters, "Bound and Approximations for Optimum Combining of Signals in the Presence of Multiple Cochannel Interferers and Thermal Noise," IEEE Transactions on Communications, vol. 51, pp. 296-307 (2003)

13. M. R. McKay, A. Zanella, I. B. Collings, and M. Chiani, "Error Probability and SINR Analysis of Optimum Combining in Rician Fading," IEEE Transactions on Communications, vol. 57, pp. 676-687 (2009)

14. J. G. Proakis, Digital Communications. McGrawHill, 4th ed. (2001). 
15. A. Jalali, R. Padovani, and R. Pankaj, "Data Throughput of CDMA-HDR a High Efficiency-High Data Rate Personal Communication Wireless System," in Proc. IEEE Vehicular Technology Conference (VTC '00), pp. 1854$1858(2000)$

16. M. K. Simon and M.-S. Alouini, Digital Communications over Fading Channels. Wiley Interscience, 2nd ed. (2004)

17. A. Papoulis and S. U. Pillai, Probability, Random Variables and Stochastic Processes. Mc Graw Hill, 4th ed. (2002)

18. I. S. Gradshteyn and I. M. Razhik, Table of Integrals, Series, and Products. Academic Press, 6th ed. (2000).

19. M. Abramowitz and I. A. Stegun, Handbook of Mathematical Functions with Formulas, Graphs, and Mathematical Tables. Dover (1970).

20. H. David and H. Nagaraja, Order Statistics. Wiley (2003). 


\section{University Library}

\section{- M M N E R VA A gateway to Melbourne's research publications}

Minerva Access is the Institutional Repository of The University of Melbourne

Author/s:

Sung, CK;Collings, IB;Elkashlan, M;Yeoh, PL

Title:

New diversity combining receivers for cooperative multiplexing in wireless multiuser relay networks

Date:

2013-09-01

\section{Citation:}

Sung, C. K., Collings, I. B., Elkashlan, M. \& Yeoh, P. L. (2013). New diversity combining receivers for cooperative multiplexing in wireless multiuser relay networks. International Journal of Wireless Information Networks, 20 (3), pp.170-182. https://doi.org/10.1007/ s10776-013-0204-X.

Persistent Link:

http://hdl.handle.net/11343/282870 